\title{
Bose-Einstein condensation phenomenology in systems with repulsive interactions
}

\author{
Oleh Savchuk, ${ }^{1}$ Yehor Bondar $\odot,{ }^{1}$ Oleksandr Stashko, ${ }^{1}$ Roman V. Poberezhnyuk, ${ }^{2,3}$ Volodymyr Vovchenko $\odot,{ }^{4,3}$ \\ Mark I. Gorenstein $\odot,{ }^{2,3}$ and Horst Stoecker ${ }^{3,5,6}$ \\ ${ }^{1}$ Physics Department, Taras Shevchenko National University of Kyiv, 03022 Kyiv, Ukraine \\ ${ }^{2}$ Bogolyubov Institute for Theoretical Physics, 03680 Kyiv, Ukraine \\ ${ }^{3}$ Frankfurt Institute for Advanced Studies, Giersch Science Center, D-60438 Frankfurt am Main, Germany \\ ${ }^{4}$ Nuclear Science Division, Lawrence Berkeley National Laboratory, Berkeley, California 94720, USA \\ ${ }^{5}$ Institut für Theoretische Physik, Goethe Universität Frankfurt, D-60438 Frankfurt am Main, Germany \\ ${ }^{6}$ GSI Helmholtzzentrum für Schwerionenforschung GmbH, D-64291 Darmstadt, Germany
}

(Received 27 April 2020; accepted 17 August 2020; published 8 September 2020)

\begin{abstract}
The role of repulsive interactions in statistical systems of Bose particles is investigated. Three different phenomenological frameworks are considered: a mean-field model, an excluded volume model, and a model with a medium-dependent effective mass. All three models are tuned to yield similar equations of state, with only minor deviations from the ideal Bose gas at small chemical potentials. Our analysis indicates, however, that these models lead to qualitatively different results for the Bose-Einstein condensation phenomenon. We discuss the different aspects of this phenomenon, namely, an onset of the Bose-Einstein condensation, particle number fluctuations, and a behavior of the Bose condensate. The obtained results can be helpful for interpreting the lattice QCD data at small temperature and large isospin chemical potential and the data on multiple pion production in high-energy nuclear collisions.
\end{abstract}

DOI: 10.1103/PhysRevC.102.035202

\section{INTRODUCTION}

The Bose-Einstein condensation (BEC) in the ideal gas of bosons was predicted many years ago [1,2] and later experimentally confirmed for cold atomic gases in magnetic traps [3-6]. This effect appears to be common for different systems of free or interacting bosons, ranging from condensed matter physics to high-energy nuclear physics and astrophysics (see, e.g., Refs. [7-14]).

The theory of the BEC phenomenon for interacting particles has been extensively discussed [15-19]. In particular, modifications of the BEC onset line (further referred to as the BEC line) due to the small repulsive interactions between particles were predicted [20-26]. Namely, an increase of the temperature of the onset of BEC due to the repulsive interactions when compared with ideal Bose gas (Id-BG) at the same density was found. However, this conclusion is not of general validity, as will be shown in the present paper. We use three different phenomenological models to describe the effects of particle repulsion in boson systems: a mean-field model, an excluded volume model, and a model with a mediumdependent effective mass. The parameters of these models are

Published by the American Physical Society under the terms of the Creative Commons Attribution 4.0 International license. Further distribution of this work must maintain attribution to the author $(s)$ and the published article's title, journal citation, and DOI. Funded by $S C O A P^{3}$. tuned to produce quantitatively similar equations of state at zero chemical potential and with only small deviations from the Id-BG. However, the properties of the BEC phenomenon appear to be very sensitive to specific features of these considered models. This fact motivates our interest to perform a comparative analysis of these models.

Most real systems have, in addition to repulsion, also attractive forces that dominate thermodynamics at low densities, producing phase diagrams with a more complex structure compared to the ones obtained here. In particular, one can observe first- and second-order phase transitions in addition to the BEC. In the phase diagram regions where such effects can be neglected, however, we expect our arguments to be true. Influence of particle interactions on the thermodynamic properties was also considered within the $S$-matrix formulation of statistical mechanics [27]. In particular, the attractive and repulsive interaction from pion-pion and other hadron-hadron scatterings were discussed in a number of works [28-31].

In addition to the behavior of the BEC line and the phase with the Bose condensate (BC), we also analyze the behavior of particle number fluctuations, as their measurements can serve as a signature of the BEC. We find qualitatively different results for all considered quantities for the BEC in the three considered models.

Our discussion is appropriate for a generic system of bosons with repulsive interactions. Nevertheless, to be specific we will refer mostly to a statistical system of $\pi$ mesons. Two arguments motivate this choice. First, recent results from lattice QCD support an existence of the pion BEC at finite isospin chemical potential [32,33], as suggested earlier by 
the chiral perturbation theory [34]. Second, the pion BEC phenomenon has a number of applications, including ultrarelativistic collisions of heavy ions $[8,9]$, the hypothetical pion stars [35-37], and the cosmic trajectory in the early universe $[32,38]$. Recently, the possibility of Bose condensation in a pion system was considered within a Skyrme-like model including both attractive and repulsive interaction terms [39].

The paper is organized as follows. Section II describes the characteristics of the BEC in the Id-BG gas. Section III presents a description of the theoretical frameworks used in the paper. In Sec. IV we present the model results for the BEC and reveal the qualitative differences obtained within the three considered models. A summary in Sec. V closes the paper.

\section{IDEAL BOSE GAS}

The pressure function of the relativistic gas in the grand canonical ensemble can be written as [40]

$$
p_{\mathrm{id}}(T, \mu)=\frac{d}{6 \pi^{2}} \int_{0}^{\infty} d k \frac{k^{4}}{\sqrt{k^{2}+m^{2}}} f_{\mathrm{k}},
$$

where the momentum distribution $f_{k}$ reads

$$
f_{\mathrm{k}}(T, \mu ; m)=\left[\exp \left(\frac{\sqrt{k^{2}+m^{2}}-\mu}{T}\right)-\eta\right]^{-1},
$$

where $\eta=1$ and $\eta=-1$ for the Bose and Fermi statistics, respectively. The classical Boltzmann approximation corresponds to $\eta=0 . m$ is the particle mass, $T$ and $\mu$ are the system's temperature and chemical potential, respectively, and $d$ is the degeneracy factor. The density of particles in the ideal gas is given by

$$
n_{\mathrm{id}}(T, \mu) \equiv\left(\frac{\partial p_{\mathrm{id}}}{\partial \mu}\right)_{T}=\frac{d}{2 \pi^{2}} \int_{0}^{\infty} d k k^{2} f_{\mathrm{k}}(T, \mu ; m) .
$$

In what follows we discuss the identical bosons in the same internal state, spin, and isospin states. Thus we keep $d=1$ for the number of internal degrees of freedom.

At fixed $T$, the particle number density (3) is a monotonously increasing function of $\mu$. For bosons, $\eta=1$, the integral in Eq. (3) reaches its maximal value at $\mu=m$. Chemical potentials values larger than $\mu=m$ are forbidden as they would lead to negative values of particle occupation numbers $f_{k}$ in some $k$ states. Note that such a restriction on $\mu$ is absent in the ideal Fermi gas with $\eta=-1$. At $\mu=m$ the total number of particles $N_{0}$ at $k=0$ may become of a macroscopic magnitude, i.e., proportional to the system's volume, $N_{0} \propto V$. In this case the particle number density $n_{0}$ in the lowest energy level, $k=0$, should be accounted separately, as an additional term in the particle number density. The total particle number density $n$ is then written as follows:

$$
n=n_{\text {id }}(T, \mu=m)+n_{0},
$$

where $n_{0} \geqslant 0$ is the density of particles with zero momentum, the so-called $\mathrm{BC}$ density.

The BEC line, $T_{c}=T_{c}(n)$, can be obtained by substituting $\mu=m$ in Eq. (3) and solving the equation with respect to $T$. For the nonrelativistic, $\left(k^{2}+m^{2}\right)^{1 / 2} \approx m+k^{2} /(2 m)$, and ultrarelativistic, $\left(k^{2}+m^{2}\right)^{1 / 2} \approx k$, approximations the solutions are explicit [40,41]:

$$
\begin{aligned}
T_{c} & \approx \frac{2 \pi}{m}\left(\frac{n}{\zeta(3 / 2)}\right)^{\frac{2}{3}} \approx 3.31 \frac{n^{2 / 3}}{m}, \quad T_{c} / m \ll 1, \\
T_{c} & \approx\left(\frac{\pi^{2} n}{\zeta(3)}\right)^{\frac{1}{3}} \approx 2.02 n^{1 / 3}, \quad T_{c} / m \gg 1,
\end{aligned}
$$

where $\zeta(x)=\sum_{n=1}^{\infty} n^{-x}$ is the Riemann zeta function with $\zeta(3 / 2) \approx 2.612$ and $\zeta(3) \approx 1.202$. The BEC in the cold lowdensity atomic gases corresponds to region $T / m<10^{-10}$, while in nuclear physics, e.g., for $\alpha$ particles, to $T / m<10^{-3}$. Thus, these physical phenomena can be accurately described within the nonrelativistic limit (5). However, the BEC of pions can happen at $T / m_{\pi} \approx 1$ and this necessitates using the relativistic formulation. The Id-BG BEC line $T_{c}(n)$ is shown by the solid line in Fig. 1(a).

The $\mathrm{BC}$ fraction,

$$
\frac{n_{0}}{n}=1-\frac{n_{\mathrm{id}}(T, \mu=m)}{n_{\mathrm{id}}\left(T_{c}, \mu=m\right)},
$$

lies between zero at the onset of the BEC at $T=T_{c}$ and unity at $T=0$. In the nonrelativistic and ultrarelativistic cases this quantity takes, respectively, the following forms:

$$
\begin{aligned}
& \frac{n_{0}}{n}=1-\left(\frac{T}{T_{c}}\right)^{\frac{3}{2}}, \quad T_{c} / m \ll 1 ; \\
& \frac{n_{0}}{n}=1-\left(\frac{T}{T_{c}}\right)^{3}, \quad T_{c} / m \gg 1 .
\end{aligned}
$$

The BC fraction $n_{0} / n$ in the Id-BG at $T<T_{c}$ is shown in Fig. 2(a).

The $\mathrm{BC}$ does not produce an additional pressure in the Id$\mathrm{BG},{ }^{1}$ thus the system pressure at the phase with $\mathrm{BC}$ equals $p_{\text {id }}(T, \mu=m)$ at $T \leqslant T_{c}$. There is also no contribution from the $\mathrm{BC}$ to the entropy density,

$$
s_{\text {id }}(T, \mu=m) \equiv\left(\frac{\partial p_{\text {id }}(T, \mu)}{\partial T}\right)_{\mu=m},
$$

whereas the energy density $\varepsilon$ at $T \leqslant T_{c}$ does get a contribution from the $\mathrm{BC}$ and reads

$$
\varepsilon=\varepsilon_{\mathrm{id}}(T, \mu=m)+m n_{0} .
$$

At $T=0$ all particles of the Id-BG are in the BC state, thus

$$
n=n_{0}, \quad p=0, \quad s=0, \quad \varepsilon=m n_{0} .
$$

A useful measure for the particle number fluctuations in the thermodynamic limit is the scaled variance:

$$
\omega=\lim _{N \rightarrow \infty} \frac{\left\langle N^{2}\right\rangle-\langle N\rangle^{2}}{\langle N\rangle},
$$

where $N$ is the total number of particles, and $\langle\ldots\rangle$ denotes the grand canonical ensemble averaging. ${ }^{2}$ The scaled variance of

\footnotetext{
${ }^{1}$ This does not necessarily apply to systems of interacting bosons.

${ }^{2}$ The finite-size effects have been discussed in Ref. [9].
} 

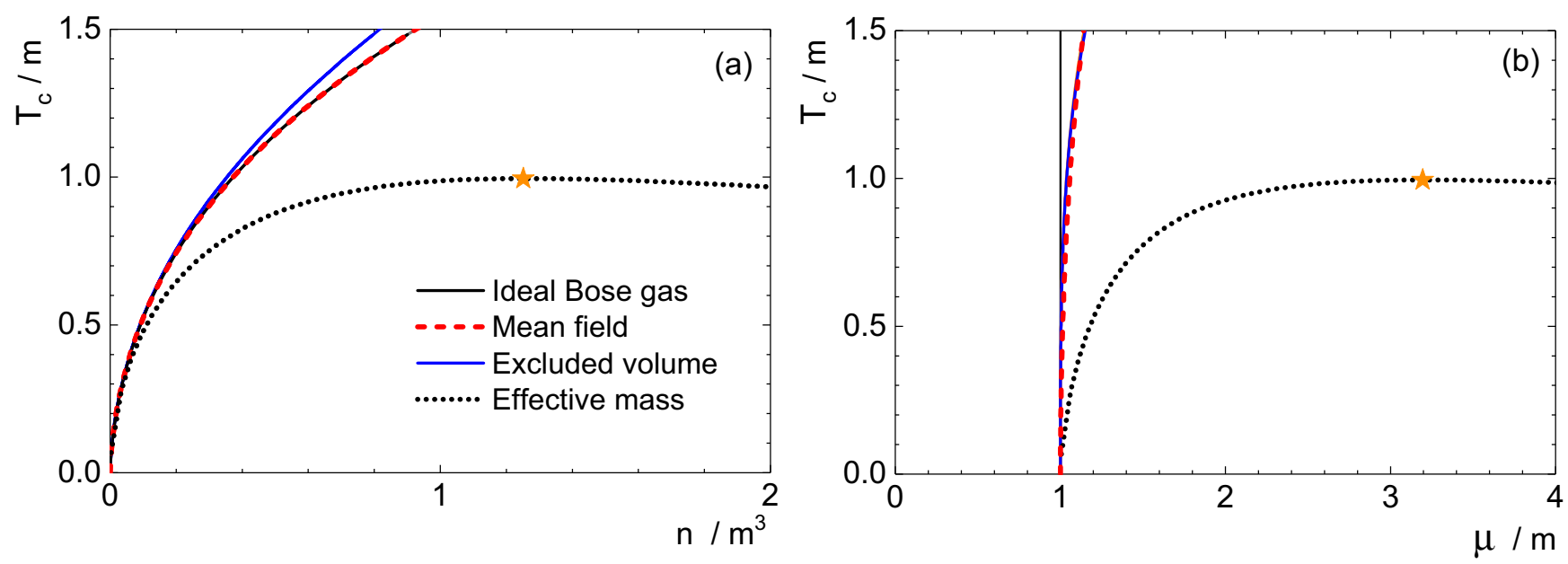

FIG. 1. (a) The BEC-lines $T_{c}=T_{c}(n)$ and (b) $T_{c}=T_{c}(\mu)$ for Id-BG, MF, EV, and EM models. The star denotes the maximal temperature $T_{c}^{\max }$ at which the BEC is possible in the EM model.

particle number fluctuations in the Id-BG is [8]

$$
\begin{aligned}
\omega & =\frac{T}{n}\left(\frac{\partial n}{\partial \mu}\right)_{T}=\omega_{\mathrm{id}}(T, \mu) \\
& =1+\frac{\eta}{2 \pi^{2} n_{\mathrm{id}}} \int_{0}^{\infty} d k k^{2}\left[f_{\mathrm{k}}(T, \mu, m)\right]^{2} .
\end{aligned}
$$

The scaled variance $\omega_{\mathrm{id}}(T, \mu)$ in the Boltzmann approximation $(\eta=0)$ equals to $\omega=1$, meaning that the particle number distribution in the classical ideal gas is given by a Poisson distribution. For the Id-BG $(\eta=1)$ the scaled variance $\omega_{\text {id }}$ is always above unity, and for the ideal Fermi gas it is always smaller than unity. $\omega_{\text {id }} \rightarrow 1$ in the $T$ - $\mu$ regions of the phase diagram where the effects of Bose and Fermi statistics are negligible, i.e., when $f_{k} \ll 1$. For the Id-BG the scaled variance (14) diverges as one approaches the BEC line:

$$
\omega_{\mathrm{id}}\left(T_{c}, \mu=m\right)=\infty
$$

Relation (15) remains valid in the phase with the BC, i.e., for all $T<T_{c}$. The scaled variance $\omega$ for the Id-BG is presented as a function of $T$ and $n$ in Fig. 3(a).

\section{MODELS OF REPULSIVE INTERACTIONS}

To address the problem of BEC in the presence of repulsive interactions we will consider three phenomenological models. As motivated in the Introduction, we shall refer to the system of $\pi$ mesons in our consideration from this point
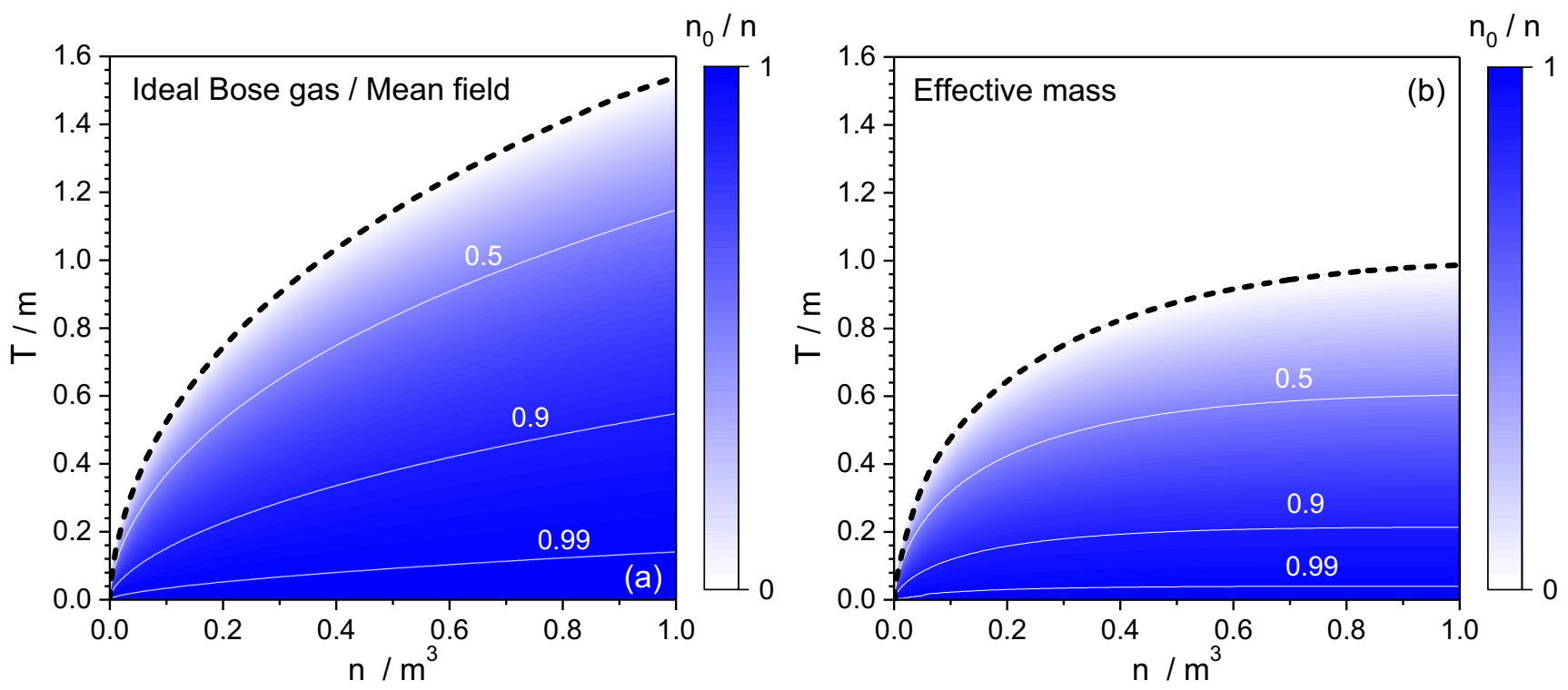

FIG. 2. The BC fraction, $n_{0} / n$, as a function on $n$ and $T$ (a) for the Id-BG and MF models, and (b) for the EM model. The line of the onset of BEC is shown by the dashed curve for each model. The result for the EV model is close to the Id-BG/MF result and, thus, is not presented. 

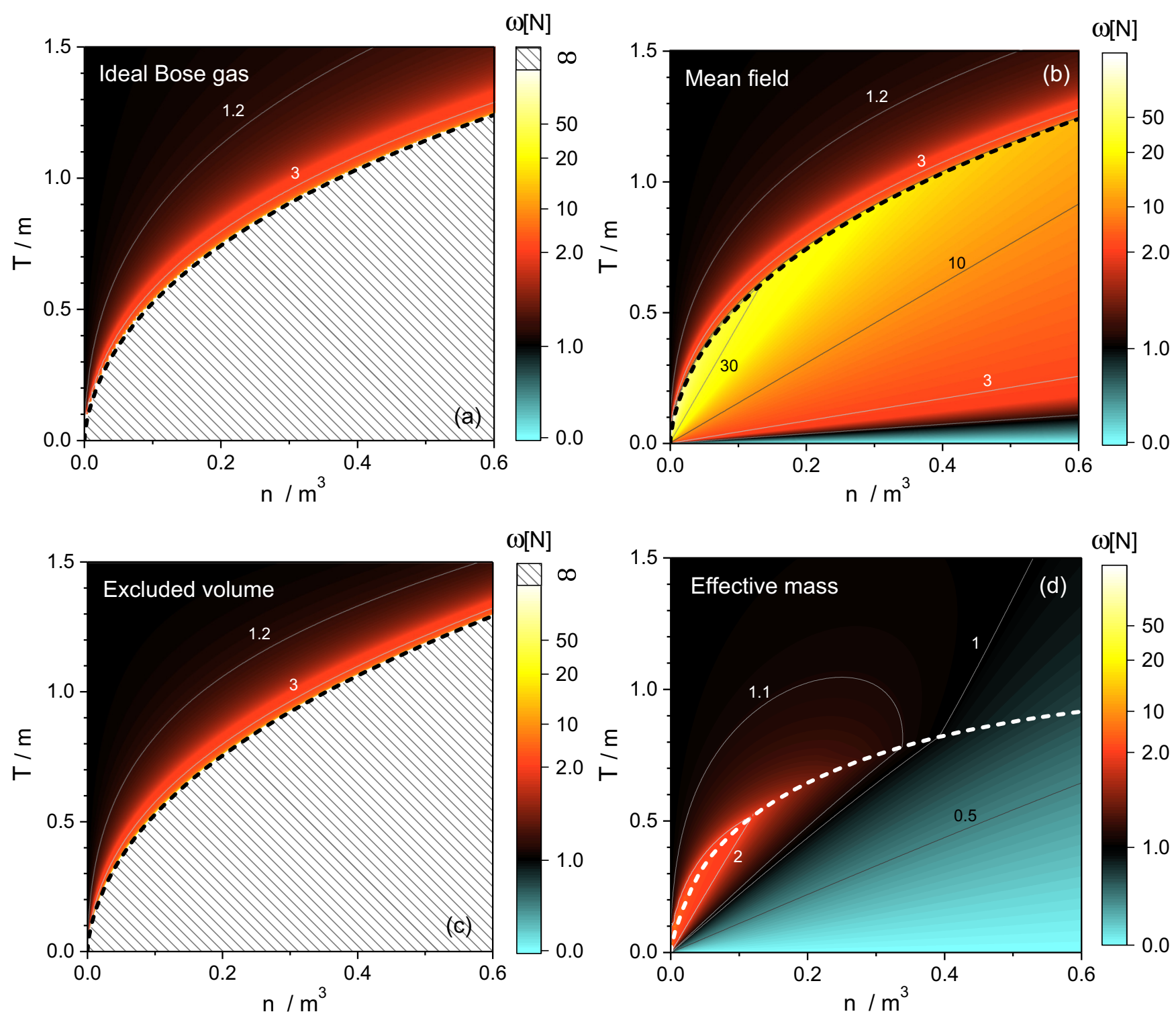

FIG. 3. The scaled variance $\omega$ of particle number fluctuations as the function of density and temperature for (a) the Id-BG, (b) the MF model, (c) the EV model, and (d) the EM model. The black color corresponds to the $(n, T)$ region where $\omega$ is close to the Poisson limit, $\omega=1$, i.e., both the Bose statistics and repulsive interaction effects are small. Regions with infinite value of $\omega$ in (a) and (c) are hatched. The line of the onset of BEC is shown by the dashed curve for each model.

on. ${ }^{3}$ The $\pi$ mesons are bosons with spin equal to zero. There are three types of pions, $\pi^{+}, \pi^{-}$, and $\pi^{0}$, with $m_{\pi^{ \pm}} \cong 140$ $\mathrm{MeV}$ and $m_{\pi^{0}} \cong 135 \mathrm{MeV}$. In the relativistic systems the number of particles is not a conserved quantity. In the case of pions, the conserved quantity is the electric charge (or, equivalently, isospin). The average value of the electric charge is regulated by the electric chemical potential $\mu_{Q}$ in the grand canonical ensemble. The chemical potentials of all three pion species are defined by $\mu_{Q}$ only: $\mu_{\pi^{+}}=\mu_{Q}, \mu_{\pi^{-}}=-\mu_{Q}$, and $\mu_{\pi^{0}}=0$. Nonzero values of $\mu_{\pi^{+}}$(or $\mu_{\pi^{-}}$) in chemically equilibrated systems are only possible for nonzero net electric charge. Chemical nonequilibrium is another possibility, where

${ }^{3}$ The obtained results, however, have general validity. fast nonequilibrium processes can produce overpopulation of all type of pions in comparison to the state of chemical equilibrium. Such a possibility has been suggested within ultrarelativistic collisions of hadrons and/or nuclei (see, e.g., Ref. [8]). In what follows we focus on the BEC of a single pion species. Therefore, we fix a single pion type with the degeneracy factor $d=1$ and particle mass $m=m_{\pi}$, keeping the notation $\mu$ for the chemical potential of that pion species.

In this section we present the formulations of the three models under consideration for phase diagram regions without BEC. In the next section we generalize these models to describe the region with a nonzero $\mathrm{BC}$ component.

Mean-field model. The first model under consideration is the thermodynamic mean-field (MF) model (see, e.g., Ref. [42] and references therein). Within the MF model the 
pressure and particle number density at given $\mu$ and $T>T_{c}$ are given by the following equations:

$$
\begin{aligned}
p(T, \mu) & =p_{\mathrm{id}}\left(T, \mu^{*}\right)+\int_{0}^{n} d n^{\prime} n^{\prime} \frac{d U\left(n^{\prime}\right)}{d n^{\prime}}, \\
n & =\left(\frac{\partial p}{\partial \mu}\right)_{T}=n_{\mathrm{id}}\left(T, \mu^{*}\right), \\
\mu^{*} & =\mu-U(n) .
\end{aligned}
$$

Here $U(n)$ is a density-dependent mean field and $\mu^{*}$ is the effective chemical potential. The purely repulsive interactions correspond to $d U / d n>0$. The MF model with

$$
U(n)=a n, \quad a>0,
$$

was applied to describe the BEC of interacting bosons in Ref. [43]. More elaborate potentials with higher powers of $n$ can be of interest at high densities, the Skyrme-like MF model with $U(n)$ describing both repulsive and attractive interactions was used to study the BEC of $\alpha$ particles in Refs. [7,44,45]. We use the simplest version of $U(n)$ in the form of Eq. (19) in the following.

The scaled variance $\omega$ (13) of particle number fluctuations in the MF model can be presented as

$$
\omega=\frac{T}{n}\left(\frac{\partial n}{\partial \mu}\right)_{T}=\frac{\omega_{\mathrm{id}}\left(T, \mu^{*}\right)}{1+\frac{a n}{T} \omega_{\mathrm{id}}\left(T, \mu^{*}\right)} .
$$

In the Boltzmann approximation $\omega_{\mathrm{id}}=1$. From Eq. (20) it then follows

$$
\omega=\left(1+\frac{a n}{T}\right)^{-1}<1,
$$

and $\omega \rightarrow 1$ at $a \rightarrow 0$. Therefore, in the classical (Boltzmann) gas the MF repulsion leads to a suppression of particle number fluctuations. A stronger repulsion (larger $a$ ) leads to the stronger suppression. In the Id-BG, on the other hand, $\omega$ is always larger than 1 due to the effects of Bose statistics.

Excluded volume model. The next approach is the excluded volume (EV) model, which describes repulsive interactions of particles in terms of their eigenvolumes. This approximation is usually used to model short-range repulsion similar to the hard-core repulsion in a classical gas of hard spheres. The EV model was generalized to include the effects of quantum statistics in Ref. [46]. It is defined by the following equations:

$$
\begin{aligned}
p(T, \mu) & =p_{\text {id }}\left(T, \mu^{*}\right), \\
n & =\left(\frac{\partial p}{\partial \mu}\right)_{T}=\frac{n_{\text {id }}\left(T, \mu^{*}\right)}{1+b n_{\text {id }}\left(T, \mu^{*}\right)}, \\
\mu^{*} & =\mu-b p_{\text {id }}\left(T, \mu^{*}\right),
\end{aligned}
$$

where $b>0$ is the classical eigenvolume parameter, which regulates the strength of repulsion. As seen from Eq. (23) the particle number density of the EV model satisfies an inequality $n<1 / b$. In the classical gas of hard spheres the parameter $b$ is identified with the second virial coefficient and is expressed through hard-core radius $r$ as $b=16 \pi r^{3} / 3$. Note, however, that in the case of quantum hard spheres the second virial coefficient suggests a temperature dependent $b$ [47], which will be addressed in future works.
The EV model scaled variance is calculated as follows [48]:

$$
\omega(T, \mu)=\frac{T}{n}\left(\frac{\partial n}{\partial \mu}\right)_{T}=(1-b n)^{2} \omega_{\mathrm{id}}\left(T, \mu^{*}\right) .
$$

The scaled variance in the EV model is suppressed by the factor $(1-b n)^{2}$ in comparison to $\omega_{\mathrm{id}}$. Therefore, for classical ideal gas, where $\omega_{\text {id }}=1$, one finds $\omega=(1-b n)^{2}<1$, i.e., the EV repulsion effects lead to a suppression of the particle number fluctuations [49].

Effective mass model. The third model that we consider is the effective mass (EM) model. A formulation of the EM model with $m^{*}=m^{*}(T)$ and $\mu=0$ was suggested in Ref. [50] (see also Refs. [51,52]). We extend the EM model to $m^{*}=m^{*}(T, \mu)$ by choosing a simple modification of the model that leads to a thermodynamically consistent description:

$$
\begin{aligned}
p(T, \mu) & =p_{\mathrm{id}}\left(T, \mu ; m^{*}\right)+\frac{\left(m-m^{*}\right)^{2}}{2 c}, \\
n(T, \mu) & =n_{\mathrm{id}}\left(T, \mu ; m^{*}\right), \\
m^{*}(T, \mu) & =m+c n_{\mathrm{id}}^{s}\left(T, \mu ; m^{*}\right),
\end{aligned}
$$

where

$$
n_{\mathrm{id}}^{s}\left(T, \mu ; m^{*}\right)=\frac{d m^{*}}{2 \pi^{2}} \int_{0}^{\infty} \frac{k^{2} d k}{\sqrt{k^{2}+m^{* 2}}} f_{\mathrm{k}}\left(T, \mu ; m^{*}\right)
$$

is the scalar density of an ideal Bose gas and $c>0$ is a model parameter.

The requirement $c>0$ leads to $m^{*}>m$, which corresponds to the repulsive interactions. The numerical value of $c$ regulates a strength of the particle repulsion. The EM model considered here resembles the Walecka model [53-55] of nuclear matter. However, the second term in the right-hand side of Eq. (26) is positive and describes the pion repulsion, whereas in the Walecka model the corresponding term is negative and it describes the attractive forces between nucleons.

The scaled variance of the particle number fluctuations is calculated as follows:

$$
\begin{aligned}
\omega & =\frac{T}{n}\left(\frac{\partial n}{\partial \mu}\right)_{T} \\
& =\frac{T}{n}\left[\frac{\partial n_{\mathrm{id}}\left(T, \mu ; m^{*}\right)}{\partial \mu}+\frac{\partial n_{\mathrm{id}}\left(T, \mu ; m^{*}\right)}{\partial m^{*}} \frac{\partial m^{*}(T, \mu)}{\partial \mu}\right] .
\end{aligned}
$$

The partial derivative $\partial m^{*}(T, \mu) / \partial \mu$ is evaluated by differentiating Eq. (28) with respect to $\mu$ and solving the resulting equation for $\partial m^{*}(T, \mu) / \partial \mu$ :

$$
\frac{\partial m^{*}(T, \mu)}{\partial \mu}=\frac{c \frac{\partial}{\partial \mu} n_{\mathrm{id}}^{s}\left(T, \mu ; m^{*}\right)}{1-c \frac{\partial}{\partial m^{*}} n_{\mathrm{id}}^{s}\left(T, \mu ; m^{*}\right)} .
$$

The expression for $\omega$ simplifies in the Boltzmann approximation $\eta=0$, i.e., when effects of quantum statistics are neglected, and by applying either nonrelativistic $\left(T / m^{*} \ll 1\right)$ 
or ultrarelativistic $\left(T / m^{*} \gg 1\right)$ limits:

$$
\begin{aligned}
& \omega=\left(1+c \frac{n}{T}\right)^{-1}, \quad T / m^{*} \ll 1, \\
& \omega=1, \quad T / m^{*} \gg 1 .
\end{aligned}
$$

Tuning the model parameters. The positive model parameters, $a, b$, and $c$ for MF, EV, and EM models, respectively, regulate the strength of repulsive interactions in all the models. At $a=b=c=0$ all the three models are reduced to the Id-BG. We fix the numerical values of the above parameters through the following considerations. At $\mu=0$ one observes a suppression of the system's pressure in comparison to the Id-BG due to the repulsion effects. These suppression effects become larger with increasing $T$, and the ratio $p(T, 0) / p_{\text {id }}(T, 0)$ thus decreases with $T$ in all three descriptions. To keep only small deviations from the Id-BG results in the whole temperature region for $\mu=0$ we fix this ratio at $m_{\pi}=135 \mathrm{MeV}, T=150 \mathrm{MeV}$ to a value

$$
\frac{p(T, \mu=0)}{p_{\text {id }}(T, \mu=0)} \cong 0.98<1,
$$

the same for all three models. Therefore, the difference between all three models is almost negligible at $\mu=0$, and their deviations from the Id-BG pressure are indeed very small at all physically reasonable $T$ values. ${ }^{4}$ The requirement (34) fixes the model parameters to

$$
\begin{aligned}
\text { MF model: } & a=0.15 m_{\pi}^{-2}, \\
\text { EV model: } & b=0.145 m_{\pi}^{-3}, \\
\text { EM model: } & c=2.21 m_{\pi}^{-2} .
\end{aligned}
$$

If $b$ is to be interpreted as the excluded volume parameter in the system of classical spheres, one finds $r \cong 0.3 \mathrm{fm}$ for the pion hard-core radius. This is consistent with the values considered in Refs. [58,59].

One comment is appropriate here. An evident intuitive expectation is that the system's pressure should increase when the interparticle repulsion is switched on. Equation (34) demonstrates the opposite behavior. This counterintuitive result comes due to a decrease of particle number density at any fixed $T$ and $\mu$ values as a consequence of the repulsive interactions. This suppression of the particle number density $n$ leads to the lower values of the pressure when compared with the corresponding values in the system of noninteracting particles at the same $T$ and $\mu$ (see Ref. [60]). The pressure in models with repulsive interaction becomes indeed higher than that of the Id-BG if they will be compared at fixed $T$ and $n$ values. Therefore, the ratio

$$
\frac{p(T, n)}{p_{\text {id }}(T, n)}>1
$$

is larger than 1 for all three models. For example, at $T=$ $110 \mathrm{MeV}$ and $n=0.06 \mathrm{fm}^{-3}$ the numerical values of the

\footnotetext{
${ }^{4}$ The QCD chiral crossover transition pseudocritical temperature at $\mu=0$ is $T_{\text {pc }} \cong 155 \mathrm{MeV}[56,57]$.
}

ratio (38) equal approximately to $1.02,1.02$, and 1.15 for MF, $\mathrm{EV}$, and EM models, respectively.

\section{BEC ANALYSIS}

MF model. In the MF model [Eqs. (16)-(19)] the BEC line corresponds to the effective chemical potential being equal to the particle's mass, $\mu^{*}=m$. Therefore, as follows from Eq. (17) the density, $n$, and temperature, $T_{c}$, at the BEC line are connected by the same equation as for the Id-BG case:

$$
n=n_{\mathrm{id}}\left(T_{c}, \mu^{*}=m\right) \text {. }
$$

Thus, the BEC line in the MF model coincides with that in the Id-BG in the $(n, T)$ plane, and it is described by the same analytic relations as in Eqs. (5) and (6). The MF model BEC line is shown in Fig. 1(a) by the dashed red ine. The BC fraction also keeps its Id-BG forms (8) and (9). This is depicted in Fig. 2(a). However, as follows from Eq. (18), the chemical potential at $T \leqslant T_{c}$ behaves as follows:

$$
\mu=m+U(n)
$$

and does not keep a constant value along the BEC line, in contrast to $\mu=m$ constant value in the Id-BG. Namely, the $\mu=\mu_{c}$ value increases along the BEC line with increasing $T_{c}$ as shown in Fig. 1(b). This is because the critical density $n=n_{c}$ [and thus the $U\left(n_{c}\right)$ contribution to $\mu_{c}$ in Eq. (40)] increases with $T_{c}$ as seen from Fig. 1(a).

At $T \leqslant T_{c}$ Eq. (17) is modified to account for the nonzero contribution from the $\mathrm{BC}$ :

$$
n=n_{\mathrm{id}}\left(T, \mu^{*}=m\right)+n_{0}, \quad T \leqslant T_{c} .
$$

This can be used to calculate the BC density $n_{0}$ as a function of $n$ and $T$. The BC density satisfies the same Eq. (7) as in the Id-BG, shown in Fig. 2(a). It leads to $0 \leqslant n_{0} \leqslant n$ with $n_{0}=0$ at $T=T_{c}$ and $n_{0}=n$ at $T=0$.

The scaled variance of particle number fluctuations is given by Eq. (20). Approaching the BEC line $\left(\mu^{*}=m\right)$ one has $\omega_{\text {id }}\left(T, \mu^{*}\right) \rightarrow \infty$ in Eq. (20) and thus

$$
\omega=\frac{T}{n}\left(\frac{d U}{d n}\right)^{-1}=\frac{T}{a n}, \quad T \leqslant T_{c} .
$$

Note that Eq. (42) remains valid at all $T \leqslant T_{c}$.

The scaled variance for the MF model is presented in Fig. 3(b). In contrast to the Id-BG case, in the MF model $\omega$ is finite both near the BEC line and inside a phase with the BC, $n_{0}>0$, changing continuously as one goes across the BEC line. Equation (20) illustrates also the requirement of thermodynamic stability of the MF model for bosons, especially on the BEC line. To fulfill an evident requirement $\omega>0$, which follows from the definition (13), one needs $d U / d n>0$. This last inequality is valid for repulsive interactions. In particular, this is valid for $U(n)$ given by Eq. (19) with $a>0$. Purely attractive interaction with $a<0$ in Eq. (19) would lead to thermodynamic instability.

EV model. The temperature $T_{c}$ on the BEC line is obtained by substituting $\mu^{*}=m$ in Eq. (23) and solving that equation with respect to $T$. In contrast to the MF model, the EV model $T_{c}(n)$ dependence does not coincide with the Id-BG result. 
At $T=T_{c}$ Eqs. (23) and (24) are transformed to

$$
\begin{aligned}
& n=\frac{n_{\text {id }}\left(T_{c}, \mu^{*}=m\right)}{1+b n_{\text {id }}\left(T_{c}, \mu^{*}=m\right)}, \\
& \mu=m+b p_{\text {id }}\left(T_{c}, \mu^{*}=m\right) .
\end{aligned}
$$

The solutions of Eqs. (43) and (44) are shown in Figs. 1(a) and 1(b), respectively. A novel feature of the EV model is the modification of the BEC line in the $(n, T)$ plane with $T_{c}^{\mathrm{EV}}(n)$ being larger than $T_{c}^{\mathrm{MF}}(n)=T_{c}^{\mathrm{id}}(n) \mathrm{BEC}$ line of the MF and Id-BG models. This modification of the BEC-line with respect to the Id-BG result, shown in Fig. 1(a) is consistent, with many other results reported for Bose gases with repulsion [20-26].

The scaled variance $\omega$ is given at $T \geqslant T_{c}$ by Eq. (25). As one approaches the BEC line $\left(\mu^{*} \rightarrow m\right)$, the Bose effects start to dominate $\omega$ since $\omega_{\mathrm{id}}\left(T, \mu^{*} \rightarrow m\right) \rightarrow \infty$, implying that particle number fluctuations start to diverge. The scaled variance remains divergent at all $T \leqslant T_{c}$ in the EV model. This behavior is similar to the Id-BG but differs from the MF model where fluctuations remain finite everywhere. The behavior of $\omega$ as a function of temperature and density in the EV model is presented in Fig. 3(c).

EM model. The phase with the BC corresponds in the EM model to a condition $\mu=m^{*}$. The BEC line is thus defined by the following equation:

$$
m^{*}\left(T_{c}\right)=m+c n_{\mathrm{id}}^{s}\left(T_{c}, \mu=m^{*} ; m^{*}\right) .
$$

The resulting BEC line $T_{c}=T_{c}(\mu)$ is shown in Fig. 1(b) by a dotted line. With $n$ calculated from

$$
n=n_{\mathrm{id}}\left(T_{c}, \mu=m^{*} ; m^{*}\right)
$$

the BEC line is shown in Fig. 1(a) as a function of particle number density, $T_{c}=T_{c}(n)$. A distinct new feature of the EM model is a decrease of the BEC line $T_{c}(n)$ as compared to the Id-BG. Therefore, the three considered models of particle repulsion show three qualitatively different possibilities for the changes of the BEC line in comparison to the Id-BG behavior, namely

$$
T_{c}^{\mathrm{EV}}(n)>T_{c}^{\mathrm{MF}}(n)=T_{c}^{\mathrm{id}}(n)>T_{c}^{\mathrm{EM}}(n) .
$$

Another interesting feature of the EM model is an absence of the BEC at large temperatures, i.e. Eq. (45) has no solutions at $T>T_{c}^{\max }$, meaning that the BEC is only possible at $T<$ $T_{c}^{\max }$. The $T_{c}^{\max }$ value is obtained by numerically analyzing Eq. (45) and it is marked by the stars in Fig. 1. Explicit expressions for $T_{c}^{\max }$ can be obtained if the non-relativistic or ultrarelativistic approximations are applied:

$$
\begin{aligned}
& T_{c}^{\max } \cong \frac{2 \pi m}{3}\left(\frac{2}{m^{2} c \zeta(3 / 2)}\right)^{2 / 3}, \quad m^{2} c \gg 1, \\
& T_{c}^{\max } \cong m\left(\frac{12}{m^{2} c}\right)^{1 / 2}, \quad m^{2} c \ll 1 .
\end{aligned}
$$

The expressions (26)-(28) for the EM model equation of state are modified in a presence of the nonzero BC density

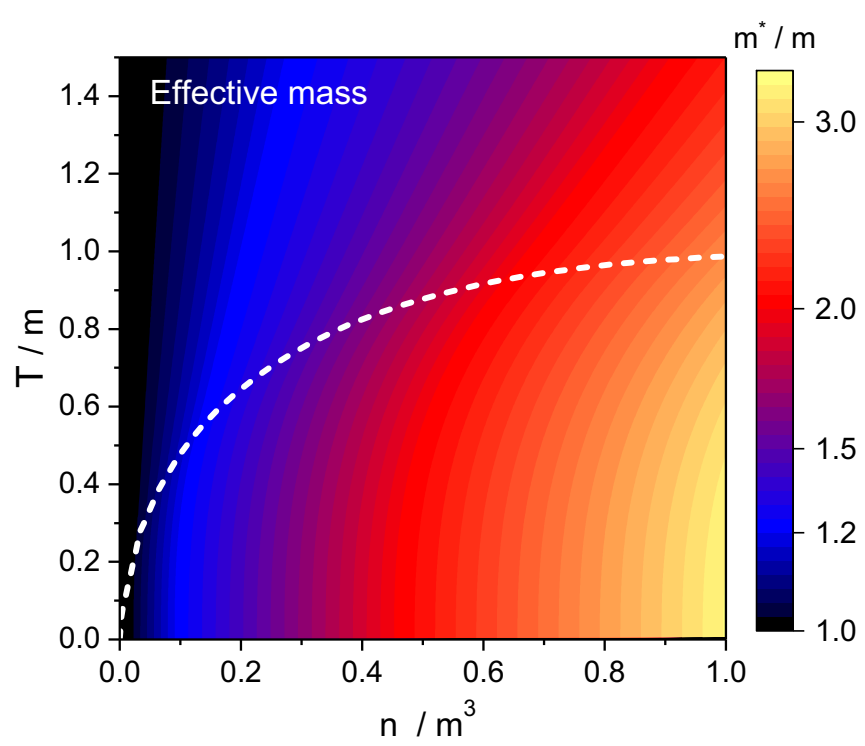

FIG. 4. Effective mass $m^{*} / m$ as a function of density and temperature for the EM model. The black color corresponds to the $m^{*}=m$ region where both the Bose statistics and repulsive interaction effects are negligible. The line of the onset of BEC is shown by the dashed curve.

$n_{0}>0$ at $T<T_{c}$. Namely,

$$
\begin{aligned}
p(T, \mu) & =p_{\mathrm{id}}\left(T, \mu ; m^{*}=\mu\right)+\frac{(\mu-m)^{2}}{2 c}, \\
n(T, \mu) & =n_{\mathrm{id}}\left(T, \mu ; m^{*}=\mu\right)+n_{0}(T, \mu), \\
n_{0}(T, \mu) & =\frac{\mu-m}{c}-n_{\mathrm{id}}^{s}\left(T, \mu ; m^{*}=\mu\right) .
\end{aligned}
$$

The BC density $n_{0}=n_{0}(n, T)$ in the EM model is presented in Fig. 2(b) whereas the behavior of the scaled effective mass $\mathrm{m}^{*} / \mathrm{m}$ presented in Fig. 4, both above and below $T_{c}$.

The scaled variance of particle number fluctuations for $T \geqslant T_{c}$ is given by Eq. (30) while for $T<T_{c}$ it is evaluated as follows:

$$
\begin{aligned}
\omega=\frac{T}{n}\left(\frac{\partial n}{\partial \mu}\right)_{T}= & \frac{T}{n}\left[\frac{\partial n_{\mathrm{id}}\left(T, \mu ; m^{*}=\mu\right)}{\partial \mu}\right. \\
& \left.-\frac{\partial n_{\mathrm{id}}^{s}\left(T, \mu ; m^{*}=\mu\right)}{\partial \mu}+\frac{1}{c}\right] .
\end{aligned}
$$

The function $\omega=\omega(n, T)$ in the EM model is presented in Fig. 3(c). We used Eq. (30) for calculations at $T \geqslant T_{c}$ and Eq. (53) for calculations at $T<T_{c}$. The scaled variance $\omega$ remains finite at all $(n, T)$ values. Furthermore, the behavior of $\omega$ is found to be continuous as one crosses the BEC line. This EM model result is similar to the MF model, but differs the Id-BG and EV results where $\omega$ is divergent for all $T \leqslant T_{c}$.

The scaled variance $\omega$ is close to unity at fixed temperature $T>T_{c}$ and small density, $n \approx 0$, in all considered models of particle repulsion. This is a region of the phase diagram where both the interaction and Bose statistics effects become negligible and the ideal gas Boltzmann approximation can be applied. However, close to the BEC line and inside the phase with the nonzero $\mathrm{BC} n_{0}>0$ the models differ qualitatively. In 
contrast to the Id-BG and EV models, one finds within the MF and EM models the finite values of $\omega$ at all temperatures and densities including those at $T \leqslant T_{c}$. These two models lead also to $\omega \rightarrow 0$ at $T \rightarrow 0$ as seen from Figs. 3(b) and 3(c).

An increase in the values of the model parameters $a, b$, and $c$ makes the repulsive effects stronger and leads to a decrease of particle number fluctuations. The maximal possible temperature of the BEC in the EM model $T_{c}^{\max }$ will become lower. The density at an onset of the BEC decreases in the EV and increases in the EM models. No qualitative changes are observed.

In the limit $T \rightarrow 0$ the entropy approaches zero, $s \rightarrow 0$, in all considered models. This is in agreement with the third law of thermodynamics. Also, all particles are located in the condensate at zero temperature, i.e., $n \rightarrow n_{0}$ as $T \rightarrow 0$, as is the case in the Id-BG. However, the pressure and energy density does depend on the specific model used. For instance, the MF model yields the following in the zero temperature limit;

$$
p=a \frac{n_{0}^{2}}{2}, \quad \varepsilon=m n_{0}+a \frac{n_{0}^{2}}{2} .
$$

The EM model gives similar expressions:

$$
p=c \frac{n_{0}^{2}}{2}, \quad \varepsilon=m n_{0}+c \frac{n_{0}^{2}}{2} .
$$

Both Eqs. (54) and (55) differ from the Id-BG result (12).

The particle number density $n$ shows a linear dependence on the chemical potential $\mu$ at $\mu>m$ in both the EM and the MF models at $T=0$, more specifically $n_{0}^{\mathrm{MF}}(\mu)=(\mu-m) / a$ [as follows from Eq. (40)] in the MF model, and $n_{0}^{\mathrm{EM}}(\mu)=$ $(\mu-m) / c$ [Eq. (52)] in the EM model. Interestingly, lattice QCD simulations at finite isospin density do exhibit the same linear increase of the isospin (pion) density in the BEC phase at $T=0$. The linear regime persists in a range $m \leqslant \mu \lesssim$ $1.4 \mathrm{~m}$, see Fig. 1 in Ref. [35]. The lattice data, therefore, can be interpreted as evidence for repulsive interactions between pions in the BEC phase. For a more quantitative analysis of the lattice data, parameters $a$ and $c$ in Eqs. (35) and (37) would have to be refitted, and possibly more involved formulations of the MF and EM models considered to reach the $\mu>1.4 m$ region. Such extensions will enable new applications, for instance to study the properties of the hypothetical pion stars [35]. These questions will be a subject of future studies.

\section{SUMMARY}

The phenomenology of the Bose-Einstein condensation (BEC) in equilibrium systems with repulsive interactions has been studied in three different models. The results from different models are compared with each other as well as with the ideal Bose gas (Id-BG) baseline. The mean-field (MF), excluded volume (EV), and effective mass (EM) models have been considered to describe the particle repulsion. The model parameters are fixed to have very similar results at $\mu=0$ for all the considered temperatures. In this region of the phase diagram, far away from the BEC onset, the three considered models demonstrate universal features of the repulsive interactions, with only minor $(\approx 2 \%)$ deviations of the system pressure from that in the Id-BG. However, the model results differ significantly in their peculiar behavior on the BEC line as well as inside the phase with a Bose condensate.

First, deviations of the BEC line $T_{c}=T_{c}(n)$ from the IdBG baseline are qualitatively different in all three considered models: $T_{c}^{\mathrm{EV}}(n)>T_{c}^{\mathrm{id}}(n)$ for the EV model, $T_{c}^{\mathrm{EM}}(n)<T_{c}^{\mathrm{id}}(n)$ for the EM model, and $T_{c}^{\mathrm{MF}}(n)=T_{c}^{\mathrm{id}}(n)$ for the MF model.

Second, essential qualitative differences are observed for the behavior of the scaled variance $\omega$ of particle number fluctuations. $\omega$ is divergent at $T \leqslant T_{c}$ in the EV model, which is similar to the Id-BG behavior. On the other hand, the values of $\omega$ remain finite and continuous at all densities $n$ and temperatures $T$ within the MF and EM models. This fact provides an opportunity to distinguish the features of particle interactions experimentally, by the measurements of particle number fluctuations.

Third, the EM model exhibits a distinctive feature: existence of the maximal temperature $T_{c}^{\max }$ above which the $\mathrm{BEC}$ does not occur. Interestingly, a similar behavior is seen in lattice QCD simulations at finite isospin, with $T_{c}^{\max } \approx$ $160 \mathrm{MeV} \approx 1.2 m_{\pi}$ [32]. There, the disappearance of the pion BEC is usually attributed to a transition to partonic degrees of freedom - a mechanism that the EM model studied here does not possess. Nevertheless, the EM model could be useful for parametrizing the QCD equation of state at finite isospin density for temperatures $T \lesssim 160 \mathrm{MeV}$, for instance by incorporating pions described by the EM model into the hadron resonance gas and by constraining the parameters by the lattice data at zero temperature.

\section{ACKNOWLEDGMENTS}

We are thankful to D. V. Anchishkin, I. N. Mishustin, and L. M. Satarov for fruitful discussions. This work is partially supported by the Target Program of Fundamental Research of the Department of Physics and Astronomy of the National Academy of Sciences of Ukraine (N 0120U100857). O.St. acknowledges the financial support by the scientific program "Astronomy and space physics" (Project No. BF19-023-01) of Taras Shevchenko National University of Kyiv. V.V. was supported by the Feodor Lynen program of the Alexander von Humboldt foundation and by the US Department of Energy, Office of Science, Office of Nuclear Physics, under Contract No. DE-AC02-05CH11231. H.St. acknowledges the support through the Judah M. Eisenberg Laureatus Chair by Goethe University and the Walter Greiner Gesellschaft, Frankfurt.
[1] S. N. Bose, Z. Phys. 26, 178 (1924).

[2] A. Einstein, Kgl. Preuss. Akad. Wiss 1, 137 (1925).
[3] M. H. Anderson, J. R. Ensher, M. R. Matthews, C. E. Wieman, and E. A. Cornell, Science 269, 198 (1995). 
[4] C. C. Bradley, C. A. Sackett, J. J. Tollett, and R. G. Hulet, Phys. Rev. Lett. 75, 1687 (1995).

[5] K. B. Davis, M. O. Mewes, M. R. Andrews, N. J. van Druten, D. S. Durfee, D. M. Kurn, and W. Ketterle, Phys. Rev. Lett. 75, 3969 (1995).

[6] F. Dalfovo, S. Giorgini, L. P. Pitaevskii, and S. Stringari, Rev. Mod. Phys. 71, 463 (1999).

[7] L. M. Satarov, M. I. Gorenstein, A. Motornenko, V. Vovchenko, I. N. Mishustin, and H. Stoecker, J. Phys. G: Nucl. Part. Phys. 44, 125102 (2017).

[8] V. V. Begun and M. I. Gorenstein, Phys. Lett. B 653, 190 (2007).

[9] V. V. Begun and M. I. Gorenstein, Phys. Rev. C 77, 064903 (2008).

[10] G. C. Strinati, P. Pieri, G. Röpke, P. Schuck, and M. Urban, Phys. Rep. 738, 1 (2018).

[11] P. Nozieres and S. Schmitt-Rink, J. Low Temp. Phys. 59, 195 (1985).

[12] Y. Funaki, T. Yamada, H. Horiuchi, G. Röpke, P. Schuck, and A. Tohsaki, Phys. Rev. Lett. 101, 082502 (2008).

[13] P.-H. Chavanis and T. Harko, Phys. Rev. D 86, 064011 (2012).

[14] I. N. Mishustin, D. V. Anchishkin, L. M. Satarov, O. S. Stashko, and H. Stoecker, Phys. Rev. C 100, 022201(R) (2019).

[15] J. I. Kapusta and C. Gale, Finite-Temperature Field Theory: Principles and Applications, 2nd ed., Cambridge Monographs on Mathematical Physics (Cambridge University Press, Cambridge, 2006).

[16] J. O. Andersen, Rev. Mod. Phys. 76, 599 (2004).

[17] A. Griffin, D. W. Snoke, and S. Stringari, Bose-Einstein Condensation (Cambridge University Press, Cambridge, 1996).

[18] S. Watabe and Y. Ohashi, Phys. Rev. A 88, 053633 (2013).

[19] S. Watabe, Acta Phys. Polon. A 135, 1222 (2019).

[20] G. Baym, J.-P. Blaizot, M. Holzmann, F. Laloë, and D. Vautherin, Phys. Rev. Lett. 83, 1703 (1999).

[21] G. Baym, J.-P. Blaizot, and J. Zinn-Justin, Europhys. Lett. 49, 150 (2000).

[22] M. Holzmann and W. Krauth, Phys. Rev. Lett. 83, 2687 (1999).

[23] M. Holzmann, G. Baym, J.-P. Blaizot, and F. Laloe, Phys. Rev. Lett. 87, 120403 (2001).

[24] K. Huang, Phys. Rev. Lett. 83, 3770 (1999).

[25] R. P. Feynman, Phys. Rev. 91, 1291 (1953).

[26] R. P. Feynman, Phys. Rev. 91, 1301 (1953).

[27] R. Dashen, S.-K. Ma, and H. J. Bernstein, Phys. Rev. 187, 345 (1969).

[28] R. Venugopalan and M. Prakash, Nucl. Phys. A 546, 718 (1992).

[29] W. Broniowski, F. Giacosa, and V. Begun, Phys. Rev. C 92, 034905 (2015).

[30] C. Fernández-Ramírez, P. M. Lo, and P. Petreczky, Phys. Rev. C 98, 044910 (2018).

[31] A. Dash, S. Samanta, and B. Mohanty, Phys. Rev. C 99, 044919 (2019).
[32] B. B. Brandt, G. Endrodi, and S. Schmalzbauer, Phys. Rev. D 97, 054514 (2018).

[33] B. B. Brandt, G. Endrodi, and S. Schmalzbauer, EPJ Web Conf. 175, 07020 (2018).

[34] D. T. Son and M. A. Stephanov, Phys. Rev. Lett. 86, 592 (2001).

[35] B. B. Brandt, G. Endrodi, E. S. Fraga, M. Hippert, J. SchaffnerBielich, and S. Schmalzbauer, Phys. Rev. D 98, 094510 (2018).

[36] M. Mannarelli, Particles 2, 411 (2019).

[37] J. O. Andersen and P. Kneschke, arXiv:1807.08951.

[38] H. Abuki, T. Brauner, and H. J. Warringa, Eur. Phys. J. C 64, 123 (2009).

[39] D. Anchishkin, I. Mishustin, and H. Stoecker, J. Phys. G 46, 035002 (2019).

[40] W. Greiner, L. Neise, and H. Stöcker, Thermodynamics and Statistical Mechanics (Springer Science \& Business Media, Berlin, 2012).

[41] L. D. Landau and E. M. Lifshitz, Statistical Physics (Pergamon Press, Oxford, 1975).

[42] D. Anchishkin and V. Vovchenko, J. Phys. G 42, 105102 (2015).

[43] Y. M. Poluektov, J. Low Temp. Phys. 186, 347 (2017).

[44] L. M. Satarov, I. N. Mishustin, A. Motornenko, V. Vovchenko, M. I. Gorenstein, and H. Stoecker, Phys. Rev. C 99, 024909 (2019).

[45] L. M. Satarov, M. I. Gorenstein, I. N. Mishustin, and H. Stoecker, Phys. Rev. C 101, 024913 (2020).

[46] V. Vovchenko, Phys. Rev. C 96, 015206 (2017).

[47] V. Vovchenko, A. Motornenko, M. I. Gorenstein, and H. Stoecker, Phys. Rev. C 97, 035202 (2018).

[48] V. Vovchenko, D. V. Anchishkin, M. I. Gorenstein, and R. V. Poberezhnyuk, Phys. Rev. C 92, 054901 (2015).

[49] M. I. Gorenstein, M. Hauer, and D. O. Nikolajenko, Phys. Rev. C 76, 024901 (2007).

[50] M. I. Gorenstein and S. N. Yang, Phys. Rev. D 52, 5206 (1995).

[51] V. V. Begun, M. I. Gorenstein, and O. A. Mogilevsky, Int. J. Mod. Phys. E 20, 1805 (2011).

[52] V. V. Begun, M. I. Gorenstein, and O. A. Mogilevsky, Ukr. J. Phys. 55, 1049 (2010).

[53] J. D. Walecka, Ann. Phys. (NY) 83, 491 (1974).

[54] B. D. Serot and J. D. Walecka, Adv. Nucl. Phys. 16, 1 (1986).

[55] R. Poberezhnyuk, V. Vovchenko, D. Anchishkin, and M. Gorenstein, Int. J. Mod. Phys. E 26, 1750061 (2017).

[56] A. Bazavov et al. (HotQCD Collaboration), Phys. Lett. B 795, 15 (2019).

[57] S. Borsanyi, Z. Fodor, J. N. Guenther, R. Kara, S. D. Katz, P. Parotto, A. Pasztor, C. Ratti, and K. K. Szabo, Phys. Rev. Lett. 125, 052001 (2020).

[58] A. Andronic, P. Braun-Munzinger, J. Stachel, and M. Winn, Phys. Lett. B 718, 80 (2012).

[59] V. Vovchenko, D. V. Anchishkin, and M. I. Gorenstein, Phys. Rev. C 91, 024905 (2015).

[60] G. D. Yen, M. I. Gorenstein, W. Greiner, and S.-N. Yang, Phys. Rev. C 56, 2210 (1997). 